\title{
Declaración de Puntarenas
}

Los Presidentes Centroamericanos, reunidos en Puntarenas, Costa Rica, del 15 al 17 de diciembre de 1990, recogiendo los anhelos de los pueblos del Istmo, declaran a Centroamérica como Región de Paz, Libertad, Democracia y Desarrollo. En este esp/ritu ratifican su compromiso con el establecimiento de una paz firme y duradera en Centroamérica; su decisión de fortalecer las relaciones de amistad, cooperación y buena vecindad; y la voluntad de perfeccionar los sistemas democráticos en la región, sobre la base de la existencia de Gobiemos electos por sufragio universal, igual, libre y secreto en todos los paises.

Expresan la imperiosa necesidad de incorporar las fuerzas irregulares a los procesos políticos y de erradicar la violencia. Declaran el compromiso con la tutela defensa y promoción de los Derechos Humanos. Reiteran su decisión de lograr la reconciliación de las sociedades centroamericanas divididas.

Reconocen que la paz en Centroamérica es una, integral e indivisible y que toda situación de violencia, donde quiera que ésta se produzca, extiende sus efectos negativos a toda la región. Por tanto, los paises del área tienen un interés legitimo y mutuo deber de solidaridad ineludible en la erradicación de toda acción violenta y terrorismo, asi como el compromiso de superar la pobreza extrema y promover el desarrollo sostenido como expresión de la profunda interdependencia, origen y destino común de los paises del istmo. En tal sentido, se comprometen a la creación de un nuevo modelo de seguridad regional.

Reconocen el impostergable requerimiento de dar impulso a una educación para la paz, y de instaurar un nuevo orden ecológico regional. Declaran su compromiso con la protección conjunta del medio ambiente reconociendo la responsabilidad compartida de los paises desarrollados en su preservación. 
Reciben el informe presentado por los ministros responsables de la Integración y Desarrollo Regional y se congratulan por los avances realizados en el cumplimiento del Plan de Acción Económico para Centroamérica (PAECA). En este orden de ideas, deciden continuar Impulsando el establecimiento de la nueva integración centroamericana, que busca fortalecer a la región como un bloque económico, para insertarla exitosamente en la economla mundial, mediante la adopción de acciones como las siguientes: la liberalización del comercio regional y extrarregional; la ejecución de una polltica regional sobre precios y abastecimiento de productos agropecuarios con el fin de garantizar la seguridad alimentaria de Cetroamérica; el apoyo al desarrollo de los sectores productivos mediante programas de modemización y reconversión; la elaboración de propuestas especlficas que conduzcan a solucionar el grave problema de la deuda que entrentan nuestros palses; y la acción regional conducente a eliminar los obstáculos discriminatorios que sufren nuestras exportaciones en otros palses.

Fundamentados en estos principios y propósitos comunes, los Presidentes convienen en lo siguiente:

1. Renovando su compromiso de fortalecer la institucionalidad democrática en el Istmo, deciden gestionar ante la Comunidad Intemacional y organismos especializados en la materia, el incremento de la cooperación dirigida a mejorar el funcionamiento de los Poderes del Estado, y particularmente a perfeccionar los mecanismos administrativos y judiciales destinados a garantizar la plena vigencia de los Derechos fundamentales del hombre.

Llaman especialmente la atención sobre su decisión de promover y fortalecer la plena vigencia de los Derechos Humanos, conviniendo en el interés de establecer programas regionales de divulgación y educación en relación con los Derechos del Hombre.

2. Expresan su profunda satisfacción por el grado de avance alcanzado en las negociaciones que lleva a cabo la Comisión de Seguridad, destinadas al logro de un acuerdo centroamericano en materia de seguridad, verificación, control y limitación de armamentos y efectivos militares, a pesar del obstáculo que significa la persistencla de las acciones armadas de los gnupos irregulares que aún operan en la región. Asimismo, muestran su complacencia por los trabajos que desarrolla dicha Comisión destinados al establecimiento de medidas de confianza entre los estados centroamericanos

Destacan los avances alcanzados en materias de tanta importancia como inventarios, factorización, desactivación de minas, desarme de 
civiles, de conformidad con las legislaciones internas, y prevención de incidentes y reconocen la cooperación que brinda la Comunidad Internacional, por mdio de las Naciones Unidas y la Organización de los Estados Americanos, para apoyar política y materialmente dichos trabajos.

Instruyen a la Comisión de Seguridad para que concluya a la brevedad el mandato que le confirieron en antigua Guatemala, para la adopción de un acuerdo de seguridad, verificación, control y limitación de armamentos y efectivos militares.

Ratifican la firme decisión de su Gobiernos de continuar desarrollando las acciones que sean necesarias para fortalecer las decisiones que emanan de las autoridades civiles legitimamente constituidas. A este respecto, destacan la importancia de que las Fuerzas Armadas y de Seguridad puedan desarrollar acciones concretas en apoyo de las autoridades civiles, para la ejecución de proyectos de beneficio social y de protección del medio ambiente.

En este contexto, los Presidentes de Costa Rica, El Salvador, Guatemala y Honduras elogian los exitosos esfuerzos del Gobierno de Nicaragua para lograr la reducción gradual de su ejército, en el espiritu de fortalecer las instituciones civiles y liberar recursos para el desarrollo económico y social.

3. Acuerdan en atención a los informes de la Comisión Ejecutiva, la creación de un Comité de Verificación de los compromisos de naturaleza política adoptados en el proceso de Esquipulas. Dicho Comité estará integrado por cinco ciudadanos centroamericanos de reconocida independencia, idoneidad y prestigio, nombrados por las Comisiones Nacionales de Reconciliación. En los próximos sesenta días, cada Comisión Nacional comunicará al respectivo gobierno a quien ha elegido para ser parte del Comité. Acuerdan, asimismo, solicitar al Secretario General de la Organización de los Estados Americanos que en consulta con la Comisión Ejecutiva, defina áreas de apoyo técnico y asesoria a este Comité.

Los Presidentes de Costa Rica, El Salvador, Honduras y Nicaragua reconocen los trabajos de la Comisión Nacional de Reconciliación de Guatemala en el desarrollo del gran diálogo nacional que busca que las aspiraciones de todos los sectores de la sociedad encuentren viabilidad mediante acuerdos y propuestas consensuales. Asimismo, destacan su efetiva labor en propiciar con el apoyo del Gobierno de Guatemala, las conversaciones entre los diversos sectores de la sociedad guatemalteca y la Unidad Revolucionaria Nacio- 
nal Guatemalteca (U.N.R.G.), para lograr que ésta abandone la lucha armada y se incorpore al proceso politico paclfico.

4. Los presidentes de Costa Rica, Guatemala, Honduras y Nicaragua, examinaron con el Presidente de El Salvador, la evolución del conflicto armado en dicho pals y el sestado actual del proceso de diálogo entre el Gobiemo de EI Salvador y la fuerza irregular armada del Frente Farabundo Martl para la Liberación Nacional.

Emiten al respecto y en relación con los últimos acontecimientos de recrudecimiento de las hostilidades, la Declaración anexa sobre la situación en El Salvador.

Los Presidentes de Costa Rica, Guatemala, Honduras y Nicaragua, conjuntamente con el Presidente de El Salvador, reiteran su decidido apoyo a los esfuerzos que en pro de la paz en El Salvador realiza el Secretario General de las naciones Unidas y expresan su firme esperanza de que puedan acordarse y ejecutarse, a corto plazo, medidas bajo supervisión internacional que aseguren el cese al fuego y su verificación por observadores de la Naciones Unidas, y el establecimiento como se ha acordado de mecanismos de garantla para el cumplimiento de los Derechos Humanos y la plena realización de la reconciliación nacional en El Salvador.

5. Analizan detenidamente las acciones desarrolladas durante el último ano por el Grupo de Observadores de las Naciones Unidas para Centroamérica (ONUCA) y destacan su aporte positivo a la situación actual en la región. Expresan su satisfacción por la resolución 675 (1990) del Consejo de Seguridad, de prorrogar por seis meses, el mandato de ONUCA.

Revisan detenidamente las recomendaciones que formulb la Comisión de Seguridad en la última reunión en Tegucigalpa, Honduras, con el objeto de fortalecer y actualizar la estructura organizativa y operativa de ONUCA, y hacer más efectiva su labor y veriticación. A este respecto, acuerdan apoyar dichas recomendaciones en el campo de comunicación, investigación, detención y coordinación e instruyen a la Comisión Ejecutiva para que, al más breve plazo, inicie consultas con la Secretarla General de la Organización de las Naciones Unidas destinadas a lograr una pronta adopción de las citadas recomendaciones, asl como para el establecimiento de un mecanismo periódico de información de las Naciones Unidas a la Comisión Ejecutiva sobre las actividades de ONUCA.

6. Para atender adecuadamente los flujos de repatriados, desplazados y refugiados que persisten en algunos palses del área, mediante 
protección y asistencia, exhortan a que se concrete el apoyo internacional para la ejecución de las propuestas y proyectos presentados por los Gobiernos convocantes en la primera reunión intemacional del Comité de Seguimiento de la Conferencia Intemacional sobre Refugiados Centroamericanos, (CIREFCA), celebrada en la sede de la Organización de las Naciones Unidas en Nueva York, el 27 y 28 de junio de este ano, y que las contribuciones anunciadas puedan ser utilizadas al más corto plazo en beneficio de estas poblaciones.

7. Coinciden en la necesidad de preservar y fortalecer las relaciones directas entre la Comunidad Europea y Centroamérica, con la participación del Grupo de los Tres, por medio de los foros institucionalizados existentes.

Asimismo formulan votos para que la próxima reunión San José VII, que tendrá lugar en Managua los días 18 y 19 de marzo de 1991, constituya un avance en la nueva etapa para profundizar el diálogo y la cooperación entre ambas regiones.

Destacan la importancia de su próxima reunión con el Presidente de México, Carlos Salinas de Gortari, deciden tomar las medidas adecuadas a efecto de intensificar las relaciones de la región con los Estados Unidos Mexicanos y expresan su deseo de que, en esa oportunidad, se puedan suscribir acuerdos que se orienten al logro de este objetivo.

Expresan su firme convicción de que las nuevas iniciativas de cooperación para la región complementarán los programas bilaterales y multilaterales existentes a la fecha, contribuyendo a incrementar los niveles de cooperación para el desarrollo e intercambio comercial.

8. Conscientes de la relación existente entre Educación y Desarrollo, reafirman su decisión de enfrentar y llenar las marcadas necesidades que aún subsisten en la región. A este respecto, respaldan las medidas adoptadas por la Coordinación Educativa y Cultural Centroamericana destinadas a ampliar y fortalecer los programas de educación preescolar; adoptar estrategias para ampliar la cobertura educativa, el incremento cualitativo de la educación y el mejoramiento de la retención y promoción educativa; impulsar los programas y políticas de educación de adultos; y promover la educación para la paz, la democracia, el desarrollo, la salud y el medio ambiente.

En particular, destacan la imperatividad de eliminar el analfabetismo en la región, así como de adecuar y renovar los curricula educacionales a las necesidades que plantea el desarrollo y a las condicio- 
nes culturales de los diferentes sectores de la población.

9. Convencidos de que la situación actual de los recursos naturales y del ambiente en la región requiere de acciones enérgicas para su presenvación, renovación y desarrollo, apoyadas por la más alta decisión polltica, reconocen la labor desarrollada por la Comisión Centroamericana de Ambiente y Desarrollo y deciden instruifla a fin de que, en un plazo de $\mathbf{9 0}$ dlas, defina las medidas necesarias para:

a. La preparación de una estrategia sobre canje de deuda externa por naturaleza, con el fin de financiar programas de desarrollo sostenido y de protección ambiental, tomando como base la iniciativa de las Américas y la deuda comercial y bilateral del Banco Centroamericano de Integración Económica (BCIE). Dicha estrategia deberá contemplar tanto la adquisición de deudas comerciales como la condonación de deudas institucionales.

b. La realización de un inventario y un censo regionales de zonas y especies que requieran de un régimen especial de protección, asl como la identificación de áreas protegidas prioritarias en zonas fronterizas.

c. La preparación de un acuerdo regional que determine el compromiso de los gobiernos centroamericanos de establecer un sistema de protección sobre las zonas y especies que sean identificadas, acuerdo que deberá ser presentado a consideración en la próxima Cumbre Presidencial.

d. La definición, en coordinación con la Comisión Ejecutiva, de una posición y una estrategia centroamericana de cara a la próxima Conferencia Mundial sobre Medio Ambiente, que tendrá lugar en Brasil en 1992.

e. La participación activa en los esfuerzos de negociación que se realizan en el Sistema de las Naciones Unidas para concluir convenciones internacionales sobre Diversidad Biológica y Cambio Climático.

f. La realización de acciones concretas destinadas a evitar que los paises controamericanos lleguen a convertirse en depósito de desechos tóxicos y sustancias nocivas para el medio ambiente y la salud, asi como su tránsito en el área, observando los senalamientos del Convenio de Basilea que regula este tipo de trasiego.

g. La identificación de las medidas necesarias para lograr una pronta y plena aplicación del Plan de Acción Forestal para Centroamérica y de los respectivos planes nacionales en cada país. 
h. La definición de mecanismos para establecer los fondos neclonalos y un fondo regional de ambiente y desarrollo, y la reallzeclón de acciones para formular la agenda centroamericana de amblente y desarrollo, en coordinación con las instanclas neclonales encargadas del tema.

Los Presidentes de El Salvador, Guatemala, Honduras y Nicaragua, compartiendo la convicción expresada por el Presidente de Costa Rlca, Rafael Angel Calderón Foumier, en el sentido de que es necesario ol establecimiento de una acción internacional concertada para entrentar los desaflos ambientales por medio de solidaridad, respeto y ammonla con la naturaleza, manifiestan con entusiasmo su respaldo a la Proclama "Hacia un Nuevo Orden Ecológico de Cooperación Internacional", emitida el 14 de diciembe de 1990 por el Presidente de Costa Rica, y formulan un llamado a la Comunidad Internacional para que apoye, mediante acciones concretas, las iniciativas contenidas en dicha Proclama en el convencimiento de que representan elementos fundamentales para asegurar a la humanidad el disfrute de su derecho a un mundo habitable.

10. Ante la amenaza que el narcotráfico representa para la estabilidad de las instituciones democráticas y el bienestar de los pueblos centroamericanos, resuelven:

a. Hacer un llamado a la comunidad internacional para intensificar el combate contra este flagelo por medio de cooperación técnica y financiera y coadyuvar efectivamente a la adopción de una política multilateral que comprenda la producción, el consumo, el procesamiento y el tráfico de drogas, asi como el control de los insumos qulmicos y los delitos financieros conexos.

b. Destacar la importancia de mejorar las condiciones económicas y sociales de los paises en vias de desarrollo, como un mecanismo que disminuya la presencia en estos de la producción o tráfico ilícito de drogas, y la necesidad de que los países industrializados tomen las medidas adecuadas para erradicar el consumo ilicito de drogas y controlar la producción y exportación de las sustancias quimicas utilizadas en su procesamiento.

c. Actuar conjunta y coordinadamente en la prevención, combate y enradicación del narcotráfico y tomar todas las medidas a su alcance para la efectividad del Acuerdo de Cooperación Regional para la Erradicación del Tráfico llícito de Drogas.

d. Crear una comisión centroamericana permanente para la erradicación de la producción, consumo, tráfico, uso indebido de 
estupefacientes y sustancias sicotrópicas y delitos conexos, especialmente la legalización de dinero y otros bienes y establecer un mecanismo regional de intercambio de información sobre estos aspectos.

e. Coordinar la vigilancia fronteriza y aduanera para prevenir el tráfico ilícito de drogas.

f. Crear un programa educativo regional contra el consumo y uso ilegal de drogas.

g. Instruir a los organismos correspondientes de cada pals para que ejecuten las acciones enumeradas y solicitar apoyo de la Comunidad Internacional y organismos especializados, con el mismo fin.

11. Convencidos de la necesidad de regular y ordenar los flujos migratorios en, hacia y desde el área, dentro de la más estricta observancia de los Derechos Humanos, acuerdan instruir a las autoridades nacionales respectivas para que, coordinadamente, tomen medidas orientadas a:

a. Armonizar la legislación y sistemas migratorios regionales.

b. Eliminar las visas en pasaportes diplomáticos y oficiales, y establecer una visa múltiple para comerciantes, empresarios, profesionales y otras categorias.

c. Establecer un formato común para los pasaportes y documentos de control migratorio.

d. Disenar un proyecto centroamericano de informática para control migratorio.

12. Manifiestan su apoyo al Parlamento Centroamericano por reconocer que será una instancia política que fortalecerá el diálogo permanente y la búsqueda del consenso regional. Instan a la Comunidad Económica Europea a hacer efectivo el apoyo ofrecido a la realización de elecciones para el Parlamento en los palses que lo requieran.

13. Reconocen y destacan que los Encuentros de Primeras Damas de Centroamérica, iniciados en 1987, brindan un significativo y relevante aporte al proceso pacificador y al desarrollo de la Agenda Social y Cultural del área, en materias tan importantes como el apoyo a la infancia afectada por la violencia armada en Centroamérica, la responsabilidad ante la mujer adolescente, y la situación de la mujer y la familia en la región. En este convencimiento. 
instruyen a sus Gobiernos para que bajo la coordinación de las Primeras Damas adopten y ejecuten, dentro de sus posibilidades, las conclusiones y recomendaciones formuladas en dichos Encuentros. En las próximas Cumbres las Primeras Damas participarán activamente en el tratamiento de la temática social.

14. Los Presidentes de Costa Rica, El Salvador, Guatemala y Honduras manifiestan su convencimiento de que los problemas económicos y sociales de Nicaragua, si no se atienden con un sentido de urgencia y con el espiritu de generosidad que la circunstancia demanda, pueden producir un grave deterioro en el proceso democrático de esa nación y en la estabilidad de la región en general. Al respecto, consideran que, para atender esos problemas, se requiere, al lado del apoyo que den los paises centroamericanos, que emule y sirva de ejemplo, un gigantesco y eficaz programa de solidaridad por parte de la Comunidad Internacional.

15. Por to expuesto, instruyen al Foro Centroamericano de Consulta y de Coordinación en Deuda Externa, integrado por los Ministros de Hacienda y los Presidentes de los Bancos Centrales de los paises del Area, para encontrar una solución, excepcional y realista, al problema de la deuda intrarregional de Nicaragua, a más tardar el 31 de marzo de 1991.

En armonia con lo expresado, instan a la Comunidad Internacional para que ejecute un Plan de Emergencia, que sea excepcional y asegure recursos adicionales, para dar contenido a las soluciones inmediatas de los problemas que Nicaragua enfrenta en los campos de deuda externa y financiamiento para la reconstrucción económica y social del país. Igualmente, solicitan al Banco Interamericano de Desarrollo y al Banco Mundial que asuman, en conjunto, la responsabilidad de coordinar y ejecutar un programa que resuelva los problemas antes mencionados.

16. Considerando la trascendencia que tiene para el desarrollo socioeconómico de la región el logro de una mayor apertura y eficiente participación en el comercio internacional, instruyen a los Ministros reponsables de la Integración y el Desarrollo Regional para que definan una política de convergencia arancelaria y aduanera centroamericana que sea congnente con las políticas de desarrollo regional de apertura externa, procurando la modernización de los instrumentos en que se ampara.

Para que los paises alcancen su uniformidad, mediante un mecanismo gradual, simultáneo y concertado, fijan los siguientes plazos: 
a. Establecimiento de los parámetros en la negociación, a más tardar el 31 de marzo de 1991.

b. Revisión general del arancel y puesta en vigencia de las tarifas en que haya consenso, a más tardar el 31 de mayo de 1991.

c. Concluir la negociación y poner en vigencia el arancel uniforme a más tardar el 31 de diciembre de 1992.

d. Aprobación de la nomenclatura arancelaria denominada "Sistema Armonizado" y el Codigo Antidumping Centroamericano, a más tardar el 31 de diciembre de 1991.

Además, instruyen al Consejo Ejecutivo del tratado General de Integración Económica que adopte las acciones pertinentes que garanticen el cumplimiento de los compromisos regionales en materia arancelaria y aduanera.

17. Considerando la urgente necesidad de desarrollar y transformar los sectores productivos de los paises centroamericanos, y como complemento de la política arancelaria que se adopte, instruyen a los Ministros Responsables de la Integración y el Desarrollo Regional, para que en un plazo máximo de tres meses y en coordinación con los sectores involucrados, elaboren un programa regional de Reconversión y Modernización de los Sectores productivos, sin perjuicio de las acciones que se llevan a cabo a nivel nacional. Este programa deberá ser ejecutado a partir de junio de 1991.

Con ese fin, hacen un llamado a los Organismos Financieros Internacionales, especialmente el Banco Interamericano de Desarrollo (BID), para que brinden el apoyo necesario para la ejecución de dicho programa.

18. Aprueban la propuesta de "Política Regional de Ciencia y Tecnologia", que elaboraron las autoridades competentes y les encomiendan completar un pertil de proyecto de inversión de desarrollo científico y tecnológico de los sectores productivos, tendiente a fortalecer su capacidad competitiva e innovación tecnológica de los sectores productivos, tendiente a fortalecer su capacidad competitiva e innovación tecnológica en congruencia con el Programa Regional de Reconversión y Modemización de estos sectores y el Programa Arancelario Regional.

19. Con el fin de facilitar el intercambio de productos agropecuarios básicos entre los países de la región y garantizar la seguridad alimentaria, instruyen a los Ministros de Agricultura para que, en 
coordinación con los Ministros Responsables de la Integración y Desarrollo Regional y demás autoridades competentes en la materia:

a. En el proceso de armonización de políticas agropecuarias, se de prioridad al tema de precios y comercialización regional de productos básicos a efecto de garantizar la satisfacción plena de las necesidades de consumo de los pueblos de la región.

b. Emprendan acciones inmediatas para la conformación de un sistema de intercambio de información de mercados.

c. Definan políticas regionales sobre el manejo de las donaciones, compra y venta de productos e insumos; importaciones concesionarias, negociaciones de excedentes y faltantes de productos agropecuarios básicos.

Los proyectos de acuerdos en esta materia deberán ser presentados en la Cumbre que tratará prioritariamente el desarrollo agropecuario.

20. Conscientes de que la deuda pública externa se ha constituido en un serio obstáculo al desarrollo económico y social de los palses centroamericanos que limita los esfuerzos para el logro de la paz y la democratización de la región y que, además, dicho problema es de responsabilidad conjunta de deudores y acreedores, hacen un llamado para que:

a. Los organismos financieros multilaterales, conjuntamente con las autoridades competentes de los países centroamericanos, establezcan urgentemente un mecanismo amplio para que readecuen los vencimientos, reduzcan el importe efectivo de los pagos y otorguen condiciones más adecuadas a las realidades económicas de los palses de la región; asl como para que modifiquen los procedimientos vigentes de gestión, aprobación y desembolso de préstamos con el fin de hacerlos más ágiles y expeditos.

b. Los paises acreedores condonen un saldo significativo de la deuda y el diferencial resultante pueda ser refinanciado en condiciones concesionales y pagado en moneda nacional para ser destinado a financiar proyectos que contribuyan a reducir la pobreza, a generar divisas y a la protección del medio ambiente.

Asimismo, instruyen a los Ministros Responsables de la Integración y el Desarrollo Regional para que, en coordinación con las autoridades competentes, establezcan un mecanismo para solucionar el problema de la deuda entre los paises de la región. 
21. Expresan su respaldo a las acciones emprendidas por los Ministros Responsables de la Integración y el Desarrollo Regional y por los Presidentes de los Bancos Centrales conducentes a la reestnucturación y revitalización del Banco Centroamericano de Integración Económica, para que, efectivamente, se constituya en el Instrumento financiero que se requiere para reactivar las economlas de los paises de la región con fundamento en los documentos titulados "Definiciones de los Representantes de los Paises en Relación con la Situación Actual y Perspectivas del BClE", fechados en Washington los dias 15 y 16 de octubre y 11 de diciembre de 1990.

En relación con to expuesto, instan al Banco Interamericano de Desarrollo para que financie a los paises de la región con el propósito de que puedan capitalizar y sanear financieramente al BCIE.

22. Manifiestan su satisfacción por la entrada en vigencia del nuevo "Sistema Regional de Pagos", destinado a dar impulso al comercio regional centroamericano: se comprometen a operarb bajo sanas normas financieras, y agradecen, especialmente el apoyo que la Comunidad Económica Europea ha brindado para su ejecución en el marco de las relaciones de cooperación con Centroamérica.

23. Expresan su complacencia a la Comunidad Económica Europea (CEE) por el otorgamiento de un tratamiento especial del Sistema Generalizado de Preferencias (SGP) a cuatro paises latinoamericanos como un apoyo en su lucha contra la producción y el tráfico de drogas. Como dicha decisión coloca en desventaja a los palses centroamericanos, instan a la CEE para que, con prontitud, extienda los mismos beneficios otorgados a esas naciones a los productos exportados por los paises de Centroamérica.

Asimismo, instruyen a los Ministros de Relaciones Exteriores para que gestionen ante esa Comunidad, la extensión de los mencionados beneficios en favor de los paises centroamericanos.

24. Considernado que, dentro del marco de cooperación entre la Comunidad Económica Europea y el Istmo centroamericano, se ha reconocido la importancia del banano en el intercambio comercial de las dos regiones y su relevancia para las economias del área, reiteran la urgencia de que, dentro de las negociaciones que al efecto se han estado desarrollando en la actual Ronda de Negociaciones multilaterales y de la estrategia del mercado único europeo de 1992, se garantice un tratamiento libre de gravámenes y de cuotas de importación a ese producto. 
25. Reiterar su satisfacción por la propuesta del Presidente de los Estados Unidos de América, George Bush, de la Iniciativa para las Américas, en la cual se cifran grandes expectativas para el desarrollo económico de Centroamérica, y se propugnan un régimen de libre comercio, la promoción de inversiones hacia la región y la adopción de mecanismos de conversión de deuda para fomentar la protección del medio ambiente.

En ese sentido instan al Congreso de los Estados Unidos de América para que apoye dicha iniciativa.

A la vez instruye a las autoridades competentes de sus paises para que establezcan un mecanismo de consulta y de coordinación, con el propósito de suscribir con los Estados Unidos de América, un tratado de justo comercio con la región, asi como para fomentar mayores flujos de inversión hacia ésta y desarrollar proyectos de impacto para la protección ambiental; todo $l o$ anterior sin perjuicio de las acciones que individualmente impulsen los paises.

26. Manifiestan su complacencia por la propuesta para la constitución de la Asociación para la Democracia y el Desarrollo de América Central (ADD), tendiente a conformar un foro para fortalecer la paz y la democracia y para contribuir al desarrollo económico de Centroamérica.

Estiman de vital importancia el papel protagónico centroamericano en la Asociación y consideran que la conducción de dicha entidad debe tener un carácter amplio, expresado mediante criterios de igualdad y representatividad de todos sus participantes.

Instruyen a la Comisión Ejecutiva integrada por los Ministros de Relaciones Exteriores y a los Ministros Responsables de la Integración y el Desarrollo Regional, para que, en forma conjunta, adopten las medidas y avancen en las gestiones destinadas a conformar la ADD.

Para que dicha iniciativa tenga efectividad y contribuya a alcanzar los citados propósitos, instan al Banco Interamericano de Desarrollo para que coordine un Grupo Consultivo Regional con la participación de organismos internacionales, agencias de cooperación y paises donantes, que sirva de apoyo a los palses centroamericanos en la creación de condiciones que faciliten la concreción de objetivos comunes en materia social, política y económica. 
27. Expresan su complacencia por la Iniciativa de Colombia, México y Venezuela para la puesta en marcha de proyectos nacionales y regionales que identifiquen y desarrollen nuevas fuentes de energla, y que contribuyan a mitigar la alta dependencia del petróleo que tienen los paises centroamericanos, agravada en perlodos de crisis.

28. Agradecen la resolución aprobada por la Asamblea General de la Organización de las Naciones Unidas, para prorrogar el Plan Especial de Cooperación para Centroamérica (PEC) por tres anos más. De la misma forma, urgen al Consejo de Administración del P.N.U.D. a aprobar la solicitud presentada por la región y se otorgue el monto solicitado que le permita continuar su funcionamiento, como apoyo fundamental para el desarrollo del área. Con el propósito de mejorar la eficacia de los programas financiados y de estrechar las relaciones del Plan con los paises del Istmo, y para dar contenido económico y técnico a las prioridades del PAECA, reiteran su instancia para que se establezca la Secretarla del Programa en un pais de la región.

29. Manifiestan su complacencia por el tratamiento favorable que han concedido las naciones amigas de México y Venezuela en el pago de la factura petrolera de los palses centroamericanos, asl como su aspiración para que las relaciones que hasta ahora se han entablado en el marco coyuntural de la crisis petrolera, se consoliden en lazos permanentes de cooperación, promoción de inversiones e intensificación del comercio.

30. Reconocen la importancia que reviste para los esfuerzos de integración, la reciente creación de la Comisión Mexicana para la Cooperación con Centroamérica, y a fin de activar las acciones conducentes a fortalecer las relaciones comerciales entre México y Centroamérica.

31. Reconocen los avances realizados en cumplimiento del PAECA, según el informe rendido con los Ministros Responsables de la Integración y el Desarrollo Regional, entre cuyos aspectos se destacan el análisis del marco jurldico de la integración realizado por la Comisión de Juristas: la entrada en vigencia del Sistema Regional de Pagos: los logros significativos, como paso previo a la suscripción de un acuerdo multilateral transitorio de libre comercio entre Honduras y el resto de los palses de la región, el inicio de programas de infraestructura flsica: la facilitación del transporte regional y de los trámites aduaneros; la armonización de políticas regionales en las áreas prioritarias del sector agropecuario; la 
elaboración de una propuesta sobre la Política y el Programa Regional en Ciencia y Tecnología; y los avances en materia de medio ambiente y desarrollo.

32. Instruyen a los Ministros Responsables de la Integración y el Desarrollo Regional para que en cumplimiento de lo establecido en el PAECA y en coordinación con las autoridades competentes, a más tardar el 31 de diciembre de 1991:

a. Formulen una política regional de turismo, fundamentada en la valorización del patrimonio natural y cultural.

b. Estudien la factibilidad de una política aérea flexible basada en la negociación.

c. Elaboren un plan para el desarrollo regional de pesca.

d. Revisen y ajusten los mecanismos que procuren la libre circulación de bienes culturales en la región.

e. Aprueben un programa regional para el fomento del sector social productivo.

f. Aprueben un programa regional en materia de asentamientos humanos y vivienda.

g. Desarrollen programa de facilitación aéreo-porturario.

Agradecen la participación activa de todos los sectores y organismos involucrados en el PAECA $\theta$ instan a la autoridades competentes a continuar los esfuerzos para el cumplimiento de dicho Plan y la presente Declaración.

33. Dada la importancia de la institucionalidad y juridicidad de la integración centroamericana, instruyen a los Ministros Responsables de este proceso para que, en la próxima Cumbre presenten a este foro una propuesta de marco normativo fundamental que legitime y garantice la seguridad jurídica necesaria para fortalecer el proceso de integración.

34. Con el fin de aumentar la presencia de los países centroamericanos en los organismos internacionales y dar mayor peso en ellos al grupo regional, acuerdan establecer mecanismos de coordinación en materia de votos y candidaturas, mediante un sisterna de estricta rotación en las postulaciones para cargos, puestos, sedes y membreslas en órganos del sistema interamericano y del sistema de las Naciones Unidas, incluyendo agencias y organismos especializados. A este respecto instruyen a la Comisión Ejecutiva y a los Ministros Reponsables de la Integración y el Desarrollo Re- 
gional para que elaboren y pongan en ejecución los mencionados mecanismos.

35. Reafirman el mandato otorgado a las diversas instancias previamente establecidas, para reglamentar, impulsar y viabilizar el cumplimiento de los acuerdos, asi como la función principal de veriticación, control y seguimiento de todos los compromisos contenidos en el Procedimiento de Guatemala y Declaraciones subsiguientes.

Acuerdan asimismo establecer el siguiente mecanismo institucional complementario destinado a sistematizar y fortalecer la organización de las Cumbres.

a. Las reuniones presidenciales se llevarán a cabo cada semestre, en los meses de junio y diciembre. Serán precedidas de una reunión preparatoria de Comisión Ejecutiva y de los Ministros Responsables de la Integración y el Desarrollo Regional a la que asistirlan Ministros y autoridades responsables de otras áreas gubenamentales, según se requiera para el desarrollo de la agenda de las Cumbres.

b. El pais sede de la Cumbre asumirá la Secretarla de la misma, por medio de su Ministerio de Relaciones Exteriores en el semestre posterior a la misma a fin de facilitar el análisis y difusión de documentos y relaciones con terceros paises y organizaciones internacionales. En este sentido, el país sede será el vocero de Centroamérica en el periodo semestral que le corresponda.

La rotación de las próximas sedes será la siguiente:

\begin{tabular}{lllll} 
& \multicolumn{3}{c}{ PERIODO } \\
ENERO - JUNIO & 1991 & COSTA RICA \\
\hline JULIO - DICIEMBRE & 1991 & EL SALVADOR \\
ENERO - JUNIO & 1992 & HONDURAS \\
JULIO - DICIEMBRE & 1992 & NICARAGUA \\
ENERO - JUNIO & 1993 & GUATEMALA
\end{tabular}

c. La Secretaria de la Cumbre transmitirá los informes, conclusiones y recomendaciones de dichas reuniones a las instancias del caso y a la Comisión Ejecutiva del Procedimiento, con el fin de lograr un efectivo seguimiento coordinado de los mismos. 
36. Acogen con beneplácito la propuesta presentada por el Grupo de Rlo durante su última reunión, celebrada en Caracas, Venezuela, para que Centroamérica participe en el Mecanismo Permanente de Diálogo y Concertación Política, y acuerdan enviar su representación conforme al orden de rotación establecido.

37. Reiteran su reconocimiento a los esfuerzos y avances que el Gobiemo de la república de Panamá ha logrado en tomo al proceso interno de consulta que se realiza en ese pais, con el propósito de determinar la posibilidad de su vinculación económica creciente al istmo centroamericano.

Igualmente, acogen con profunda satisfacción las claras manifestaciones del Gobierno de Panamá de incrementar su participación en las instancias políticas y económicas centroamericanas y de reforzar su vinculación con los organismos regionales.

38. Reconociendo el trascendental aporte brindado a la causa de la paz y la distensión Internacional por el Presidente de la Unión de Repúblicas Socialistas Soviéticas, Mijail Gorbachov, Premio Nobel de la Paz 1990, y en el convencimiento de que su visita a Centroamérica contribuiria a destacar la importancia de la causa de la paz y la democracia en la región y a impulsarla decididamente, acordaron invitarle a que visite oficialmente Centroamérica.

39. Los Presidentes de Costa Rica, El Salvador, Honduras y Nicaragua ante la próxima terminación de mandato del presidente de Guatemala, Marco Vinicio Cerezo Arévalo, manifestar su reconocimiento por el papel fundamntal que ha desempenado en favor de la paz regional y destacan que fue su iniciativa la que reunió a los cinco mandatarios centroamericanos en Esquipulas. Agradecen su permanente dedicación y esfuerzo a lo largo del proceso de Esquipulas II, cuya importancia en la consecución de los objetivos acordados coloca hoy a los centroamericanos frente a halagadoras perspectivas para el logro de la paz firme y duradera en la región.

40. Conscientes del fundamental papel que corresponde a las actividades agrícolas, forestales y pecuarias en la vida económica y social de los paises centroamericanos, y convencidos de la necesidad de impulsar su desarrollo en todos los ordenes, convienen en que una proxima Cumbre sea dedicada preferentemente al sector agropecuario.

41. Acuerdan reunirse de nuevo en el transcurso del primer semestre de 1991 en la República de El Salvador. 
42. Los Presidentes de El Salvador, Guatemala, Honduras, Nicaragua y Panamá, expresan su profundo agradecimiento al Presidente de Costa Rica, Rafael Angel Calderón Fournier, al Gobierno y al pueblo costarricense por la hospitalidad y las atenciones brindadas que contribuyeron significativamente al éxito de la reunión.

Puntarenas, 17 de diciembre de 1990.

\section{Rafael Angel Calderón Fournier \\ Presidente de la República de Costa Rica}
Marco Vinicio Cerezo Arévalo Presidente de la República de Guatemala

Alfredo Cristiani Burkard Presidente de la República de El Salvador

Rafael Leonardo Callejas Romero Presidente de la República de Honduras

Violeta Barrios de Chamorro

Presidente de la República de Nicaragua

OBSERVADOR INVITADO

Guillermo Endara Galimany

Presidente de la República de Panamá 